\title{
Extent of field change in colorectal cancers with BRAF mutation
}

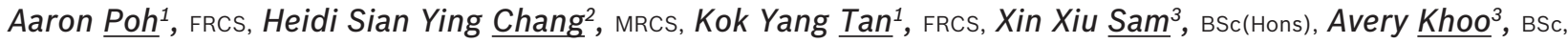 \\ Shoa Nian $\underline{\mathrm{Choo}}^{4}$, BSc, Min En $\underline{\mathrm{Nga}}^{4}$, FRCPath, Wei Keat Wan ${ }^{5}$, FRCPath
}

INTRODUCTION Sporadic colorectal cancers with BRAF mutations constitute two distinct subgroups of colorectal cancers. Recent studies have linked the presence of the BRAF mutation to a familial inheritance pattern. This was a proofof-concept study that aimed to examine: (a) the extent of field change in sporadic colorectal cancers with BRAF mutation; and (b) the extent of resection margins required and the pattern of DNA mismatch repair protein loss in these tumours. METHODS Eight microsatellite instability-high tumours with positive BRAF mutation from an existing histopathological database were selected for BRAF mutation and mismatch repair protein analysis.

RESULTS All the resection margins were negative for BRAF mutation. Three tumours had loss of MLH1 and PMS2 expressions, and five tumours had no protein loss. Six peritumoral tissues were negative and one was positive for BRAF mutation.

CONCLUSION The results suggest that any early field change effect is restricted to the immediate vicinity of the tumour and is not a pan-colonic phenomenon. Current guidelines on resection margins are adequate for BRAF mutation-positive colorectal cancers. Any suggestion of a hereditary link to these tumours is likely not related to germline BRAF gene mutations. The pattern of protein loss reinforces previous findings for the two subgroups of BRAF mutation-positive colorectal cancers.

Keywords: BRAF mutation, colorectal cancer

\section{INTRODUCTION}

Colorectal cancers can be divided into several groups based on their molecular characteristics. These characteristics are underpinned by different pathways of tumorigenesis (Table I). The earliest known pathways are the chromosomal instability (familial adenomatous polyposis [FAP]) and DNA mismatch repair (MMR) (hereditary non-polyposis colorectal cancer [HNPCC]) pathways. ${ }^{(1-3)}$ The serrated pathway was subsequently noted after the observation that a subgroup of hyperplastic polyps behaved in a manner similar to adenomatous polyps, resulting in an analysis of serrated polyps and their associated pathway. ${ }^{(1)}$

Further work on the classification of colorectal cancers focused on additional molecular characteristics for further delineation. Jass ${ }^{(2)}$ hypothesised in 2007 that there are five separate subgroups of colorectal cancers based on multiple molecular characteristics. Sporadic colorectal cancers with BRAF mutations constitute two separate subgroups out of the five possible subgroups of colorectal cancers according to this classification. These two subgroups were the focus of our study.

The first subgroup is made up of colorectal cancers characterised by high levels of DNA methylation at CpG islands (CIMP-high), methylation of the MutL homolog 1 (MLH1) gene, presence of BRAF mutation and high levels of microsatellite instability (MSI-H). These are chromosomally stable tumours and make up $12 \%$ of colorectal cancers. ${ }^{(3-8)}$ The second subgroup is made up of colorectal cancers characterised by CIMP-high, partial methylation of the MLH1 gene, presence of BRAF mutation, and stable microsatellite instability (MSS) or low levels of microsatellite instability (MSI-L). Tumours in the second group are also chromosomally stable and make up $8 \%$ of colorectal cancers. ${ }^{(1-8)}$ The two subgroups of colorectal cancers share some common characteristics, namely the presence of BRAF mutation, high levels of CIMP methylation, an element of MLH1 gene methylation (either partial or full) and an element of microsatellite instability (MSI-L or MSI-H). ${ }^{(1-8)}$

Microsatellite instability is also seen in a third subgroup of colorectal cancers, namely in HNPCC or Lynch syndrome. In contrast to the above two subgroups, HNPCC tumours are hereditary in nature but do not have BRAF mutations, are CIMPnegative and always show MSI-H. HNPCC is characterised by germline mutations in the genes that encode DNA MMR proteins. More than $90 \%$ of these mutations occur in the genes encoding for MLH1 and MutS homolog $2(\mathrm{MSH} 2)$, with the remainder occurring in the MSH6 and PMS1 homolog 2 (PMS2) genes. Due to the inherent germline mutations present, there is a generalised field change, and the entire colon and rectum are at risk for development of tumours. ${ }^{(1,2,9)}$

The group of BRAF mutation-positive colorectal cancers was the subject of the present study. The presence of BRAF mutation in the presence of MSI-H status has been touted as a negative predictor for germline MMR mutations and a possible exclusion criterion for germline testing. However, some recent

${ }^{1}$ Department of General Surgery, Khoo Teck Puat Hospital, ${ }^{2}$ University Surgical Cluster, National University Hospital, ${ }^{3}$ Histopathology Translational Research Group, Department of Pathology, Singapore General Hospital, ${ }^{4}$ Department of Pathology, National University Hospital, ${ }^{5}$ Department of Pathology, Singapore General Hospital, Singapore Correspondence: Dr Chang Sian Ying Heidi, Resident, University Surgical Cluster, National University Hospital, 5 Lower Kent Ridge Road, Singapore 119074. heidi.chang@mohh.com.sg 
Table I. Classification of colorectal cancers based on molecular characteristics.

\begin{tabular}{|c|c|c|c|c|c|}
\hline Characteristic & $\begin{array}{l}\text { Chromosomal instability (FAP) } \\
\text { pathway }\end{array}$ & $\begin{array}{l}\text { Mismatch repair (HNPCC) } \\
\text { pathway }\end{array}$ & \multicolumn{2}{|c|}{ Serrated pathway } & Hybrid pathway \\
\hline Heredity & $\begin{array}{l}\text { Sporadic and hereditary (FAP } \\
\text { syndrome) }\end{array}$ & $\begin{array}{l}\text { Hereditary only (HNPCC } \\
\text { syndrome) }\end{array}$ & \multicolumn{2}{|c|}{ Sporadic and hereditary } & Sporadic only \\
\hline MSI status & MSS & MSI-H & MSI-H & MSI-L & MSS or MSI-L \\
\hline $\begin{array}{l}\text { Chromosomal } \\
\text { instability }\end{array}$ & Present & Absent & Absent & Absent & Present \\
\hline BRAF mutation & No & No & Yes & Yes & No \\
\hline KRAS mutation & Yes & Yes/No & No & No & Yes \\
\hline CIMP status & Negative & Negative & High & High & Low \\
\hline MLH1 status & Normal & Mutated & Methylated & Partial methylation & Normal \\
\hline
\end{tabular}

CIMP: CpG island methylator phenotype; FAP: familial adenomatous polyposis; HNPCC: hereditary non-polyposis colorectal cancer; KRAS: V-Ki-ras2 Kirsten rat sarcoma viral oncogene homolog; MLH1: MutL homolog 1; MSI: microsatellite instability; MSI-H: microsatellite instability-high; MSI-L: microsatellite instability-low; MSS: microsatellite stable

population-based studies have suggested a familial tendency for colorectal cancers with BRAF mutation or CIMP-high status, suggesting a possible new cancer family syndrome. ${ }^{(10-12)}$ There is no direct evidence of the extent of the field change around this group of tumours that can provide support for either theory, and hence, the adequacy of current resection margins is questionable. Current resection margin standards of a $5-\mathrm{cm}$ margin proximal and distal to the tumour are derived from research on a separate group of tumours arising from the chromosomal instability pathway (i.e. the FAP pathway). There is a need to exclude the presence of a generalised field change and, if it is excluded, to determine the extent of the local field change in sporadic BRAF mutation-positive tumours.

Mutation of the BRAF gene occurs early in the tumorigenesis pathway leading to these two subgroups of colorectal cancers. ${ }^{(1-4,13}$ Using this knowledge as a foundation for this study, BRAF mutations can be used as a surrogate marker for detection of early field change in apparently normal mucosa that can potentially lead to tumour formation in the future. The concept of field change, also known as field cancerisation, was first mooted in a study by Slaughter et al in 1953. ${ }^{(14)}$ Field change is described as a 'process whereby cells in a particular tissue or organ are transformed such that genetically altered but histologically normal-appearing cells precede the development of neoplasia or coexist with malignant cells, irrespective of clonality'. ${ }^{(15)}$ The essence of field change is the existence of normal-looking cells or tissue that harbours genetic alterations predisposing them to overt malignant transformation. Multiple mechanisms of field change have been proposed, ${ }^{(16}$ including the polyclonal model, monoclonal expansion model and mitochondria mutation model. The concept of field change can explain the phenomenon of multiple synchronous tumours occurring within the same organ or the development of metachronous tumours in a short time interval. ${ }^{(15,17)}$

The present proof-of-concept study aimed to examine: (a) the extent of field change in sporadic colorectal cancers with BRAF mutation, with the hypothesis that the field change effect, as manifested by the presence of BRAF mutation, would be a localised field change extending for an undetermined distance around the tumour; and (b) the extent of the resection margins required and the pattern of MMR protein loss in these tumours.

\section{METHODS}

This was a retrospective study. Colorectal tumours from an existing histopathological database were filtered and eight MSI-H tumours with positive BRAF mutation were selected for further testing. Paraffin-fixed slides from the tumour and the proximal and distal margins were extracted from the database and used for testing.

Tumour blocks, adjacent non-tumour blocks and margin blocks were selected for analysis. Histological review was performed to ensure the absence of histological evidence of a tumour. Adjacent normal mucosa was defined as histologically normal mucosa at a distance of at least $5 \mathrm{~mm}$ from the tumour. Each of the aforementioned formalin-fixed paraffin-embedded (FFPE) blocks were divided into ten sections of $5 \mu \mathrm{m}$ in thickness and deparaffinisation using standard xylene with the automated Ventana system was performed. DNA extraction was performed using the Qiagen FFPE Kit and DNA concentration was assessed using the Nanodrop analyser. Minimum DNA amounts of $300 \mathrm{ng}$ were required for BRAF analysis. BRAF mutational analysis was performed using the Entrogen KRAS/BRAF Mutation Analysis Kit (v1.2). This kit uses allele-specific real-time polymerase chain reaction (PCR) and detects the $\mathrm{V} 600 \mathrm{E}$ mutation of exon 15 of the BRAF gene. It may also detect some BRAF V600K mutations as V600E mutations. The BRAF V600E mutation accounts for a majority of BRAF mutations. The assay demonstrates a limit of detection of up to $1 \%$ sensitivity.

The presence of four MMR proteins, namely MLH1, MSH2, MSH6 and PMS2, was assessed by immunohistochemical staining of the tumour tissue. The Leica BOND-MAX Autostainer Immunohistochemistry ${ }^{\mathrm{TM}}$ using the Bond polymer refine detection technique was used in our study. Appropriate internal and external controls were ensured together with the test samples. Reading was done using nuclear positivity and negativity as the categorical results. No family history fulfilling the Revised Bethesda Guidelines ${ }^{(9)}$ was found during a review of the case notes.

\section{RESULTS}

There were three right-sided colon tumours, four left-sided colon tumours and one rectal tumour in the eight selected BRAF mutation-positive colorectal cancers. All eight tumours were 
solitary tumours, with no polyps in the resected specimens. The median tumour size was 4.5 (range 3.5-8.0) cm, with six out of eight tumours having N2 staging (Table II). All tumours were positive for the BRAF V600E mutation.

The median proximal resection margin was 6.3 (range 1.5-18.5) $\mathrm{cm}$ and the median distal resection margin was 7.8 (range 4.5-22.0) cm. All eight proximal margin specimens tested negative for BRAF mutation. Seven distal margin specimens tested negative for BRAF mutation and one specimen had an inconclusive result due to suboptimal DNA. Six peritumoral specimens tested negative for BRAF mutation, one specimen had an inconclusive result due to suboptimal DNA, and the one specimen that tested positive for BRAF mutation had a V600E mutation located on exon 15. In five tumour specimens, all of the four MMR proteins tested were present. The remaining three tumour specimens showed the absence of both MLH1 and PMS2.

Two specimens failed the BRAF assay due to poor DNA quality, one from the distal resection margin and the other from peritumoral tissue. DNA extraction and PCR for BRAF mutation assay were repeated for these two specimens, but were not successful. The likely reason for the failure was that the processes of fixation and storage had affected the integrity of the DNA in these specimens. In view of the retrospective nature of the project, additional tissue was not available for further retesting. Table II summarises the results of the eight specimens selected.

\section{DISCUSSION}

The presence of BRAF mutation was used as a marker for early field change in this study. As BRAF mutation occurs relatively early in the tumorigenesis pathway, it has utility as a marker for field change in microscopically normal tissue. Notably, there was an absence of BRAF mutation at the proximal and distal resection margins in our group of specimens from tumours with proven BRAF mutation. In addition, seven of eight specimens also had no BRAF mutation at the peritumoral site. As mutation of the BRAF gene is thought to occur early on in the tumorigenesis pathway, our results suggest that any early field change effect is restricted to the immediate vicinity of the tumour and is not a pan-colonic phenomenon, as manifested by the absence of BRAF mutation at the resection margins. This conclusion was based on the assumption that the field change occurs in a continuous fashion emanating from the primary tumour. Although we recognise that the field change effect can occur at multiple discontinuous areas throughout the colon, we would still expect to observe a local field change effect around the tumour, if such an effect is present. The absence of a field change effect is supported by the fact that only one peritumoral specimen tested positive for BRAF mutation in our study. The absence of BRAF mutation at the resection margins also suggested that current guidelines on the extent of resection margins are likely to be adequate.

A proper assessment of the extent of any local field change effect requires tissue sampling in a circumferential four-quadrant fashion at predetermined distances from the tumour edge. That extent of tissue sampling was not possible within the limitations of this archival retrospective study, which used previously sampled tissue, and hence only a single-site sampling was performed. We acknowledge that the presence of any discontinuous pan-colonic phenomena would require the above sampling steps to be carried out in the vicinity of the adenomatous polyps, in addition to that of the primary tumour. This is based on the assumption that these polyps may potentially transform into malignant tumours in the future, and hence an area of local field change effect can be expected.

Recent observations have unveiled a possible hereditary link for BRAF mutations and/or CIMP-high colorectal tumours. ${ }^{(10-12)}$ The results of this study do not completely support the possibility of a hereditary link, but they do show that further studies into this link are necessary. The absence of BRAF mutation in the peritumoral tissues in our study, despite observational studies suggesting a hereditary link, might be explained as these patients having an unknown underlying genetic predisposition to developing BRAF mutation but not a germline BRAF mutation. The other explanation is that there is incomplete penetrance or variable expressivity of the underlying unknown genetic predisposition, such that the expression of BRAF mutation occurs at random areas of the colon instead of being a continuous pan-colonic phenomenon. We recognise that the genetic linkage may be related to DNA hypermethylation instead of BRAF mutation and that the lack of CIMP status testing in our study weakens any conclusion drawn from our findings regarding the hereditary nature of BRAF mutation or CIMP-high tumours. ${ }^{(18-20)}$

The use of BRAF as a marker for field change for BRAF mutation-positive colorectal tumours is a relatively new idea and has some limitations. BRAF mutation occurs early in the tumorigenesis pathway leading to the colorectal cancers found in these two subgroups. It is not likely for BRAF mutation to occur late in the tumorigenesis pathway with a separate gene mutation replacing it in the early part of the pathway. The acquisition of BRAF mutation is an essential early step in the pathway that leads on to cell senescence and inhibition of apoptosis..$^{(1-4,13)}$ This allows the cell to subsequently accumulate further genetic mutations, leading to the development of colorectal cancer. As all the tumours in our study were positive for BRAF mutations, it is reasonable to expect that any further field change in the surrounding tissue would also undergo the same pathway, with BRAF mutation occurring early in the pathway. Hence, the absence of BRAF mutation can be taken to be the absence of a field change effect. As mentioned earlier, the lack of testing for CIMP status in this study could be identified as a potential weak point, as DNA hypermethylation is a crucial intermediate step in the pathway leading to partial or complete MLH1 promoter methylation and corresponding partial or complete loss of expression of MLH1. We recognise that the absence of both BRAF mutation and hypermethylation in surrounding normal tissues is stronger evidence of the absence of a field change effect. The extent and pattern of hypermethylation in the surrounding tissues may also be potentially different between these two subgroups of tumours. Nonetheless, the absence of BRAF mutation in this proof-of-concept study is sufficient proof to suggest the absence of a local field change effect around the primary tumour. 
Table II. Results of BRAF mutational and MMR protein status analyses.

\begin{tabular}{|c|c|c|c|c|c|c|c|c|}
\hline \multirow[t]{2}{*}{ Specimen } & \multirow{2}{*}{$\begin{array}{l}\text { Tumour type, size, } \\
\text { stage }\end{array}$} & \multirow[t]{2}{*}{ Location } & \multirow{2}{*}{$\begin{array}{l}\text { Distance from } \\
\text { tumour }(\mathrm{cm})\end{array}$} & \multirow[t]{2}{*}{ BRAF mutation } & \multicolumn{4}{|c|}{ MMR protein status } \\
\hline & & & & & MLH1 & MSH2 & MSH6 & PMS2 \\
\hline \multirow[t]{4}{*}{ A } & \multirow{4}{*}{$\begin{array}{l}\text { Sigmoid colon, } 4.0 \mathrm{~cm} \text {, } \\
\text { pT4a N2 }\end{array}$} & Tumour & NA & Present & Present & Present & Present & Present \\
\hline & & Peritumoral & NA & Present & - & - & - & - \\
\hline & & Proximal margin & 1.5 & Absent & - & - & - & - \\
\hline & & Distal margin & 22.0 & Absent & - & - & - & - \\
\hline \multirow{3}{*}{ B } & \multirow{3}{*}{$\begin{array}{l}\text { Proximal transverse } \\
\text { colon, } 5.0 \mathrm{~cm} \text {, pT3 NO }\end{array}$} & Peritumoral & NA & Absent & - & - & - & - \\
\hline & & Proximal margin & 18.5 & Absent & - & - & - & - \\
\hline & & Distal margin & 5.0 & Absent & - & - & - & - \\
\hline \multirow[t]{3}{*}{ C } & \multirow{3}{*}{$\begin{array}{l}\text { Descending colon, } \\
8.0 \mathrm{~cm}, \mathrm{pT} 4 \mathrm{a} \mathrm{N} 2\end{array}$} & Tumour & NA & Present & Absent & Present & Present & Absent \\
\hline & & Peritumoral & NA & Absent & - & - & - & - \\
\hline & & Proximal margin & 7.0 & Absent & - & - & - & - \\
\hline \multirow[t]{4}{*}{ D } & \multirow{4}{*}{$\begin{array}{l}\text { Sigmoid colon, } 6.5 \mathrm{~cm} \text {, } \\
\text { pT4a N2 }\end{array}$} & Tumour & NA & Present & Present & Present & Present & Present \\
\hline & & Peritumoral & NA & Inconclusive* & - & - & - & - \\
\hline & & Proximal margin & 4.5 & Absent & - & - & - & - \\
\hline & & Distal margin & 4.5 & Absent & - & - & - & - \\
\hline \multirow[t]{4}{*}{ E } & \multirow{4}{*}{$\begin{array}{l}\text { Sigmoid colon, } 4.0 \mathrm{~cm} \text {, } \\
\text { pT4a N2 }\end{array}$} & Tumour & NA & Present & Present & Present & Present & Present \\
\hline & & Peritumoral & NA & Absent & - & - & - & - \\
\hline & & Proximal margin & 3.0 & Absent & - & - & - & - \\
\hline & & Distal margin & 8.5 & Absent & - & - & - & - \\
\hline \multirow[t]{3}{*}{$\mathrm{F}$} & \multirow{3}{*}{$\begin{array}{l}\text { Caecum, } 5.0 \mathrm{~cm}, \text { pT3 } \\
\text { N2 }\end{array}$} & Tumour & NA & Present & Absent & Present & Present & Absent \\
\hline & & Peritumoral & NA & Absent & - & - & - & - \\
\hline & & Proximal margin & 7.0 & Absent & - & - & - & - \\
\hline \multirow{3}{*}{ G } & \multirow{3}{*}{$\begin{array}{l}\text { Rectum, } 3.5 \mathrm{~cm}, \mathrm{pT} 4 \mathrm{a} \\
\mathrm{N} 1\end{array}$} & Peritumoral & NA & Absent & - & - & - & - \\
\hline & & Proximal margin & 5.5 & Absent & - & - & - & - \\
\hline & & Distal margin & 7.0 & Absent & - & - & - & - \\
\hline \multirow[t]{4}{*}{$\mathrm{H}$} & \multirow{4}{*}{$\begin{array}{l}\text { Hepatic flexure, } \\
3.5 \mathrm{~cm}, \text { pT3 N2 }\end{array}$} & Tumour & NA & Present & Present & Present & Present & Present \\
\hline & & Peritumoral & NA & Absent & - & - & - & - \\
\hline & & Proximal margin & 11.0 & Absent & - & - & - & - \\
\hline & & Distal margin & 5.0 & Absent & - & - & - & - \\
\hline
\end{tabular}

*Due to suboptimal DNA. MLH1: MutL homolog 1; MMR: DNA mismatch repair; MSH2: MutS homolog 2; MSH6: MutS homolog 6; NA: not available; PMS2: PMS1 homolog 2, mismatch repair system component

MMR protein loss was noted in three patients, who all had similar patterns of protein loss, namely of both MLH1 and PMS2. This is compatible with the pattern of protein loss reported in the literature for BRAF mutation-positive MSI-H tumours, namely loss of MLH1 expression secondary to hypermethylation of the MLH1 gene promoter. ${ }^{(1-5)}$ The tumours of these three patients belonged to the first subgroup of colorectal cancers. In contrast, over $90 \%$ of the protein loss pattern in hereditary Lynch syndrome is that of both MLH1 and MSH2. ${ }^{(2,3,9)}$

There was no loss of MLH1 in five patients. For this group, it was likely that the colorectal cancers arose from a different pathway leading to the second subgroup of colorectal cancers. There was only partial methylation of the MLH1 gene, with associated decreased expression of the MLH1 gene. Nonetheless, as there was still partial gene expression in these specimens, MLH1 protein was low but still detectable, and the degree of microsatellite instability was decreased, leading to MSI-L or MSS status.
The first limitation of our study was its retrospective nature, which only allowed sampling of tissues from existing formalinfixed slides. Any field change effect is potentially circumferential around the tumour. However, the area available for sampling was only from one sector of the tumour, while other peritumoral sectors were unavailable for sampling. It was possible that other sectors harboured BRAF mutations, as manifested by one of the specimens demonstrating a BRAF mutation in the peritumoral tissue. The small sample size of the study was also one possible reason that only one specimen demonstrated peritumoral BRAF mutation. A similar argument can also be made regarding sampling at the resection margins. However, the specimen slides at the resection margins did encompass the entire circumference of the colon or rectum, and hence the risk of sampling error was smaller. The second limitation was the lack of data on CIMP status and MSI status, which precluded an examination of the link between BRAF mutation, CIMP status and MSI status in this group of BRAF mutation-positive cancers. ${ }^{(1-4,6,6,7,19,20)}$ 
As a proof-of-concept study, we have demonstrated the absence of BRAF mutation in surrounding tissues from a small sample size of formalin-fixed slides. The next step would be to demonstrate the presence of BRAF mutation, DNA methylation (CIMP status) and MSI status from random sampling at various locations in the colon and from non-cancerous polyps in patients with a diagnosed BRAF mutation-positive and/or CIMP-high colorectal cancer.

In conclusion, BRAF mutation-positive colorectal cancers are likely to be sporadic in nature. Any suggestion of a hereditary link to these tumours is likely not related to germline BRAF gene mutations. Current guidelines for 5 -cm resection margins appear to be adequate for colorectal cancers harbouring BRAF mutations. The pattern of protein loss observed in our specimens reinforces the finding that there are two subgroups of BRAF mutation-positive colorectal cancers.

\section{REFERENCES}

1. Noffsinger AE. Serrated polyps and colorectal cancers: new pathway to malignancy. Annu Rev Pathol 2009; 4:343-64.

2. Jass JR. Classification of colorectal cancer based on correlation of clinical, morphological and molecular features. Histopathology 2007; 50:113-30.

3. Kang GH. Four molecular subtypes of colorectal cancer and their precursor lesions. Arch Pathol Lab Med 2011; 135:698-703.

4. Weisenberger DJ, Siegmund KD, Campan M, et al. CpG island methylator phenotype underlies sporadic microsatellite instability and is tightly associated with BRAF mutation in colorectal cancer. Nat Genet 2006; 38:787-93.

5. Rasuck CG, Leite SM, Komatsuzaki F, et al. Association between methylation in mismatch repair genes, V600E BRAF mutation and microsatellite instability in colorectal cancer patients. Mol Biol Rep 2012; 39:2553-60.

6. de Maat MF, Narita N, Benard A, et al. Development of sporadic microsatellite instability in colorectal tumours involves hypermethylation at methylated-in- tumor loci in adenoma. Am J Pathol 2010; 177:2347-56.

7. Zlobec I, Bihl M, Foerster A, Rufle A, Lugli A. Comprehensive analysis of $\mathrm{CpG}$ island methylator phenotype (CIMP)-high, -low, and -negative colorectal cancers based on protein marker expression and molecular features. J Pathol $2011 ; 225: 336-43$

8. Stefanius K, Ylitalo L, Tuomisto A, et al. Frequent mutations of KRAS in addition to BRAF in colorectal serrated adenocarcinoma. Histopathology 2011; 58:679-92.

9. Umar A, Boland R, Terdiman JP, et al. Revised Bethesda Guidelines for hereditary nonpolyposis colorectal cancer (Lynch syndrome) and microsatellite instability. J Natl Cancer Inst 2004; 96:261-8.

10. Young J, Barker MA, Simms LA, et al. Evidence for BRAF mutation and variable levels of microsatellite instability in a syndrome of familial colorectal cancer. Clin Gastroenterol Hepatol 2005; 3:254-63.

11. Frazier ML, Xi L, Zong J, et al. Association of the CpG island methylator phenotype with family history of cancer in patients with colorectal cancer. Cancer Res 2003; 63:4805-08.

12. Vandrovcova J, Lagerstedt-Robinsson K, Påhlman L, Lindblom A. Somatic BRAFV600E mutations in familial colorectal cancers. Cancer Epidemiol Biomarkers Prev 2006; 15:2270-3.

13. Chan TL, Zhao Z, Leung SY, Yuen ST; Cancer Genome Project. BRAF and KRAS mutations in colorectal hyperplastic polyps and serrated adenomas. Cancer Res 2003; 63:4878-81.

14. Slaughter DP, Southwick HW, Smejkal W. Field cancerization in oral stratified squamous epithelium; clinical implications of multicentric origin. Cancer 1953; 6:963-8.

15. Dakubo GD, Jakupciak JP, Birch-Machin MA, Parr RL. Clinical implications and utility of field cancerization. Cancer Cell Int 2007; 7:2.

16. Chai H, Brown RE. Field effect in cancer-an update. Ann Clin Lab Sci 2009; 39:331-7.

17. Hawthorn L, Lan L, Mojica W. Evidence for field effect cancerization in colorectal cancer. Genomics 2014; 103:211-21.

18. Samowitz WS, Albertsen H, Herrick J, et al. Evaluation of a large, populationbased sample supports a CpG island methylator phenotype in colon cancer. Gastroenterology 2005; 129:837-45.

19. Pinheiro M, Ahlquist T, Danielsen SA, et al. Colorectal carcinomas with microsatellite instability display a different pattern of target gene mutations according to large bowel site of origin. BMC Cancer 2010; 10:587.

20. Yamauchi M, Morikawa T, Kuchiba A, et al. Assessment of colorectal cancer molecular features along bowel subsites challenges the conception of distinct dichotomy of proximal versus distal colorectum. Gut 2012; 61:847-54. 